\title{
Application of Probabilistic and Nonprobabilistic Hybrid Reliability Analysis Based on Dynamic Substructural Extremum Response Surface Decoupling Method for a Blisk of the Aeroengine
}

\author{
Bin Bai, ${ }^{1,2}$ Wei Zhang, ${ }^{1,2}$ Botong Li, ${ }^{1,2}$ Chao Li, ${ }^{3}$ and Guangchen Bai ${ }^{3}$ \\ ${ }^{1}$ College of Mechanical Engineering \& Applied Electronics Technology, Beijing University of Technology, Pingleyuan 100, \\ Chaoyang District, Beijing 100022, China \\ ${ }^{2}$ Beijing Key Laboratory for the Nonlinear Vibration and Strength of Mechanical Structures, Beijing University of Technology, \\ Pingleyuan 100, Chaoyang District, Beijing 100022, China \\ ${ }^{3}$ School of Energy and Power Engineering, Beihang University, Beijing 100191, China
}

Correspondence should be addressed to Bin Bai; baibin@126.com and Wei Zhang; sandyzhang0@yahoo.com

Received 23 September 2016; Accepted 9 January 2017; Published 28 March 2017

Academic Editor: Giacomo Frulla

Copyright (C) 2017 Bin Bai et al. This is an open access article distributed under the Creative Commons Attribution License, which permits unrestricted use, distribution, and reproduction in any medium, provided the original work is properly cited.

For the nondeterministic factors of an aeroengine blisk, including both factors with sufficient and insufficient statistical data, based on the dynamic substructural method of determinate analysis, the extremum response surface method of probabilistic analysis, and the interval method of nonprobabilistic analysis, a methodology called the probabilistic and nonprobabilistic hybrid reliability analysis based on dynamic substructural extremum response surface decoupling method (P-NP-HRA-DS-ERSDM) is proposed. The model includes random variables and interval variables to determine the interval failure probability and the interval reliability index. The extremum response surface function and its flow chart of mixed reliability analysis are given. The interval analysis is embedded in the most likely failure point in the iterative process. The probabilistic analysis and nonprobabilistic analysis are investigated alternately. Tuned and mistuned blisks are studied in a complicated environment, and the results are compared with the Monte Carlo method (MCM) and the multilevel nested algorithm (MLNA) to verify that the hybrid model can better handle reliability problems concurrently containing random variables and interval variables; meanwhile, it manifests that the computational efficiency of this method is superior and more reasonable for analysing and designing a mistuned blisk. Therefore, this methodology has very important practical significance.

\section{Introduction}

In practical engineering, many uncertainties need to be considered when analysing the reliability of a complex structure. Typically, probability theory and fuzzy set are used to address uncertainty in the traditional method, and the probabilistic model has become the most common and effective method for handling uncertainty. However, the probabilistic model and fuzzy model require more data to define the parameters of the probability distribution function. Furthermore, probabilistic reliability is very sensitive to the tail of the probability density function, which plays a key role in the calculation; therefore, a small error in the data may lead to a large error in the structural reliability calculation $[1,2]$. The probabilistic model has certain limitations for the complexity structures, which has continued to increase with the progress of science and technology. In addition, only a certain range of uncertainties can be obtained instead of their probabilistic distribution. Some scholars believe that the probabilistic model cannot be accurately defined with too little data. Currently, the interval model can be used to describe uncertainties; the nonprobabilistic method is used to analyse the structure. In 1994, Ben-Haim [1] proposed the concept of nonprobabilistic reliability based on the convex set 
model and measured reliability through the maximum extent of the uncertainty for a system. Ben-Haim and Elishakoff [3] proposed a measurable method for the nonprobabilistic concept; he thought that nonprobabilistic reliability belonged to a certain range of nondeterministic parameters; that is, the reliability index is an interval rather than a specific variable.

The models mentioned in this paper can be used to describe uncertainties. Some uncertain parameters have sufficient statistical data that can be used to establish the probabilistic distribution function, while other uncertainties can be described only by a range of their variables due to a lack of statistical information; only convex sets or interval variables can be used to describe the latter uncertainties. In this case, the probabilistic method is not suitable for the structure, and the existing statistical information cannot be used by the nonprobabilistic method. Therefore, a satisfactory result cannot be obtained with only one type of model. Nevertheless, for a problem that contains both probabilistic and nonprobabilistic variables, the probabilistic and nonprobabilistic hybrid reliability method can take full advantage of the known information to achieve an effective analysis. Many scholars have studied probabilistic and nonprobabilistic hybrid reliability analysis (HRA) [4-16].

For instance, Qiu et al. developed a hybrid of probabilistic and nonprobabilistic reliability theory, with the structural uncertain parameters as interval variables when statistical data are found insufficient. Then they proposed a new reliability model to improve the evaluation of probabilistic and nonprobabilistic hybrid structural systems. In addition, he presented a recognition method for the main failure modes using a five-bar statically indeterminate truss structure and an intermediate complexity wing structure to demonstrate that the new model was more suitable for analysis and design than the probabilistic model. A new hybrid reliability model that contained randomness, fuzziness, and nonprobabilistic uncertainty based on the structural fuzzy random reliability and nonprobabilistic set-based models was presented in [6]. Furthermore, based on a residual strength model, the fatigue reliability was evaluated using hybrid uncertain parameters in [7]. Furthermore, Fang et al. converted the interval variables and fuzzy variables into random variables based on the maximum entropy principle and effectively analysed the reliability in the situation that random variables, fuzzy variables, and interval variables coexist in a problem. The branch-and-bound method for the probabilistic reliability analysis of structural systems was combined by Wang et al. with the nonprobabilistic branchand-bound method for determining the dominant failure modes of an uncertain structural system; meanwhile, the compatibility of the classical probabilistic model and the interval-set model was discussed by them, verifying the physical meaning of safety measures. In [11], a modified fuzzy interval perturbation method was proposed for predicting a hybrid uncertain temperature field involving both interval and fuzzy parameters in material properties and boundary conditions. In addition to this, An et al. introduced the truncated probability reliability model, the fuzzy random reliability model, and the nonprobabilistic interval reliability model to present a new hybrid reliability index to evaluate structural hybrid reliability based on the random-fuzzyinterval theory. Jiang et al. employed the probabilistic and nonprobabilistic convex model methods to address two cases for the uncertainty domain and the failure surface; they analysed cracked structures, addressed the difficulties in epistemic uncertainty modelling, adopted the scaled boundary finite element method to calculate the stress intensity, and developed the response surface to solve the hybrid reliability model. Furthermore, Han et al. adopted the response surface technique to compute the interval of the failure probability of the structure based on the probability-interval hybrid uncertainty.

There are other methods for HRA [17-24]; for example, [17] developed a new HRA technique for structures with multisource uncertainties that contained randomness, fuzziness, and nonprobabilistic boundedness. Hurtado and Alvarez proposed a Monte Carlo method for probability and interval approaches for reliability analysis; meanwhile, they discussed the use of Monte Carlo methods for both reliability and interval analysis from the point proposed representation. In addition to this, a mixed perturbation Monte Carlo method with a mixture of random and interval parameters was presented by Gao et al., and, meanwhile, three theories or methodologies, namely, the Taylor expansion, matrix perturbation theory, and random interval moment method, were combined to develop expressions for the mean value and standard deviation of random interval structural responses. Besides, a hybrid probabilistic model was presented in [19] to solve the fatigue reliability problems of steel bridges. Considering the parameter characteristics of the plot of stress against the number of cycles to failure ( $S-N$ curve), Wu et al. proposed a hybrid probabilistic and interval computational scheme to robustly assess the stability of engineering structures. Also, based on $S-N$ approach and fracture mechanics approach, Xue et al. assessed a tension leg platform using hybrid probabilistic and nonprobabilistic models. In addition to this, the HRA was also used in the nuclear industry; for example, Ibáñez-Llano et al. presented a new approach for estimating the exact probabilistic quantification results by combining Monte Carlo simulation with the truncation limits of the binary decision diagram approach in the nuclear industry.

Other researchers optimized structures and studied their sensitivity through probabilistic and nonprobabilistic hybrid reliability methodology as well [25-31]. For example, Luo and Zhang investigated an adhesive bonded steel-concrete composite beam with probabilistic and nonprobabilistic uncertainties and mathematically formulated the reliability-based optimization, incorporating mixed reliability constraints as a nested problem. Pedroni and Zio considered the model of an aircraft with a twin-jet engine, including 21 inputs and 8 outputs; meanwhile, they propagated the aleatory uncertainty described by probability distributions using MCS and solved the numerous optimization problems related to the propagation of epistemic uncertainty using interval analysis. Besides, Liu et al. developed a hybrid uncertainty model and used an efficient decoupling strategy to solve the nesting optimization problem to obtain an equivalent single-layer optimization model. Furthermore, based on active learning 
kriging, Yang et al. investigated HRA. The nonprobabilistic set-theory convex model was combined with the classical probabilistic approach to optimize structures exhibiting random and uncertain-but-bounded mixed uncertainties in [31].

In other applications as well, for example, [32-39], Xia and $\mathrm{Yu}$ proposed the change-of-variable interval stochastic perturbation method to predict the interval of the response probability density function and the response confidence interval of a hybrid uncertain structural-acoustic system with random and interval variables. Meanwhile, Chen et al. presented a hybrid stochastic interval perturbation method for the unified energy flow analysis of coupled vibrating systems. In addition to this, combining with a backpropagation neural network, Peng et al. investigated reliability analysis based on the hybrid uncertainty reliability mode, in the process of their research, the random variables and interval variables were used as the input layer of the neural network, and the response variables were obtained through the output layer. Furthermore, Fan and Zhang used a convex model to simulate the uncertainties of isolated structural parameters and used a random model to simulate the uncertainties of the seismic input. An interval analysis for parameter identifications was presented by Zhang et al. to address both measurement noise and model uncertainty. Xu et al. developed a model called inexact two-stage fuzzy chance-constrained programming for handling multiple uncertainties associated with solid waste management systems. Meanwhile, they structured the safety margin equation to analyse the fatigue reliability of the system by adopting the "stress-strength" theory. Also, Drugowitsch and Pouget used probabilistic and nonprobabilistic approaches for the neurobiology of perceptual decision-making.

For an aeroengine working in a complicated high temperature, high pressure, and high rotational speed environment, significant information is available as uncertain parameters, such as rotational speed and temperature. However, other uncertain parameters, such as the coefficient of thermal conductivity and the coefficient of expansion, lack sufficient data. Therefore, a new type of probabilistic and nonprobabilistic HRA is proposed based on the traditional probabilistic model and nonprobabilistic model. Unlike the probabilistic and nonprobabilistic hybrid models investigated by the abovementioned scholars, the methodology presented in this paper is connected with the improved hybrid interface substructural component modal synthesis method in [40]. This new method is called the probabilistic and nonprobabilistic hybrid reliability analysis based on dynamic substructural extremum response surface decoupling method (P-NP-HRA-DS-ERSDM) and is used to analyse the blisk of aeroengine. The reliability problems are better resolved by the hybrid model containing both random and interval variables.

\section{Basic Theory of P-NP-HRA-DS-ERSDM}

For designing and analysing engineering structures, random variables and interval variables need to be included simultaneously. Therefore, a probabilistic and nonprobabilistic

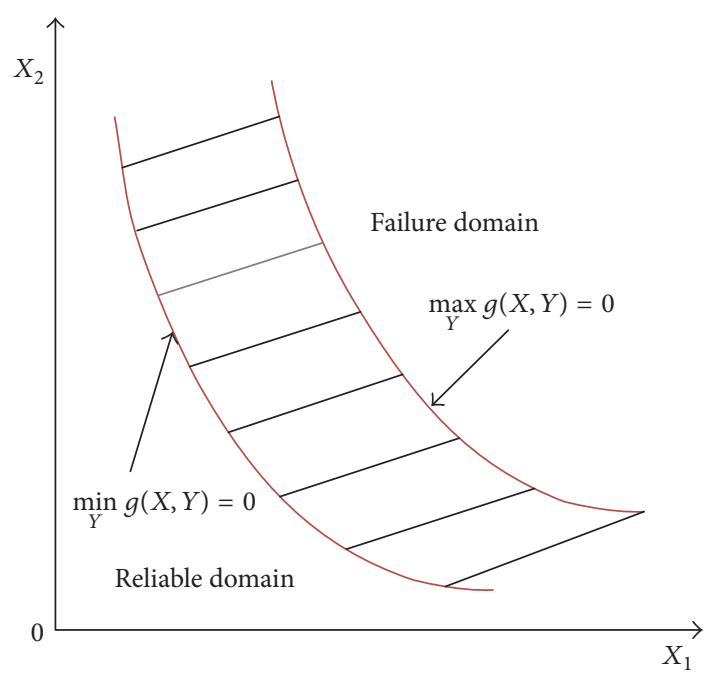

FIgURE 1: The region of extreme postural belt.

hybrid reliability model needs to be established to fully describe the actual situation of the structures.

Assume that the random variable of the system is $X=$ $\left(x_{1}, x_{2}, \ldots, x_{m}\right)$ and the interval variable is $Y=\left(y_{1}, y_{2}, \ldots\right.$, $\left.y_{n}\right)$. Then, the expression of the performance function is written as $Z=g(X, Y)$, and the failure probability is defined as $P_{f}=\operatorname{Pr}\{g(X, Y) \leq 0\}$.

After introducing the interval variable $Y$, the limit state surface function $g(X, Y)=0$ is no longer the unique surface in the space $X$, but it is a belt body that consists of two boundary surfaces, that is, $\max _{Y} g(X, Y=0)$ and $\min _{Y} g(X, Y=0)$, as shown in Figure 1.

Therefore, the failure probability $P_{f}$ has upper and lower boundaries:

$$
\begin{aligned}
& P_{f}^{\min }=\operatorname{Pr}\left\{\max _{Y} g(X, Y) \leq 0\right\}, \\
& P_{f}^{\max }=\operatorname{Pr}\left\{\min _{Y} g(X, Y) \leq 0\right\} .
\end{aligned}
$$

The reliability index $\beta$ is not a specific value but an interval (i.e., $\beta \in\left[\beta^{L}, \beta^{R}\right]$ ), where $\beta^{L}$ and $\beta^{R}$ are the maximum and the minimum reliability indexes, respectively.

Then the following two optimization problems can be solved: the maximum and the minimum reliability indexes of the limit state belt can be obtained.

$$
\begin{aligned}
\beta^{R}=\min & \|U\| \\
\text { s.t. } & \max _{Y} G(U, Y)=0, \\
\beta^{L}=\min & \|U\| \\
\text { s.t. } & \max _{Y} G(U, Y)=0,
\end{aligned}
$$

where $G(\cdot)$ is the performance function of $X$ transformed into standard normal space and $U(\cdot)$ is standard normal space. 
The failure probabilities of the maximum and the minimum values are expressed as

$$
\begin{aligned}
& P_{f}^{\min }=\Phi\left(-\beta^{R}\right), \\
& P_{f}^{\max }=\Phi\left(-\beta^{L}\right)
\end{aligned}
$$

In engineering applications, the maximum failure probability of the structure is often the one of most concern, and it has significant reference value for engineering and technical staff. Therefore, the maximum failure probability is taken as the measurement for the structural reliability in this paper.

When using the traditional multilevel nested algorithm (MLNA), the computation efficiency is very low for some mechanical parts such as a complex aeroengine working in a poor environment. Thus, the decoupling method proposed in [41] is utilized in this paper, and the proposed methodology is called P-NP-HRA-DS-ERSDM. The modal and vibration response of a blisk are analysed using this method. According to the actual conditions, the cross terms are ignored, and the response surface function can be represented as follows:

$$
\widetilde{g}(X, Y)=a_{0}+\sum_{i=1}^{m} b_{i} X_{i}+\sum_{j=1}^{n} c_{j} Y_{j}+\sum_{i=1}^{m} d_{i} X_{i}^{2}+\sum_{j=1}^{n} e_{j} Y_{j}^{2},
$$

where $a_{0}, b, c, d$, and $e$ are $(2 m+2 n+1)$ undetermined coefficients in the quadratic polynomial.

The sample centre point $(\bar{X}, \bar{Y})$ is continually updated in the solution process. For the first time, the iteration of $(\bar{X}, \bar{Y})$ is located in $\left(\mu_{X}, Y^{c}\right)$, where $\mu_{X}$ is the mean of the random variable $X, Y^{c}$ is the median of the interval variable $Y$, and the coordinates are given in (6). The other $(2 m+2 n)$ sample points are selected around the centre point, as shown in Figure 2.

$$
\begin{aligned}
& \bar{X}_{i} \pm k_{x} \cdot \sigma_{X_{i}}, \quad i=1,2, \ldots, m, \\
& \bar{Y}_{j} \pm k_{y} \cdot Y_{j}^{r}, \quad j=1,2, \ldots, n,
\end{aligned}
$$

where $\sigma_{X_{i}}$ is the standard deviation of the random variable $X_{i}, Y_{j}^{r}$ is the radius of the interval variable $Y_{i}$, and $k_{x}, k_{y}$ are the coefficients of the sample points for the random variable and the interval variable, respectively.

The value of the original performance function is calculated at each sample point; then, undetermined coefficients of the response surface function are solved.

Combining the probabilistic and nonprobabilistic hybrid reliability model with (3), the approximate mixed reliability model is constructed as follows:

$$
\begin{aligned}
\widetilde{\beta}^{L}=\min _{U} & \|U\| \\
\text { s.t. } & \max _{Y} \widetilde{G}(U, Y)=0,
\end{aligned}
$$

where $\widetilde{G}$ is the approximate limit state function in the space $U$.

P-NP-HRA-DS-ERSDM is used to solve the probabilistic and nonprobabilistic HRA of the blisk, and the specific iterative process is as follows.

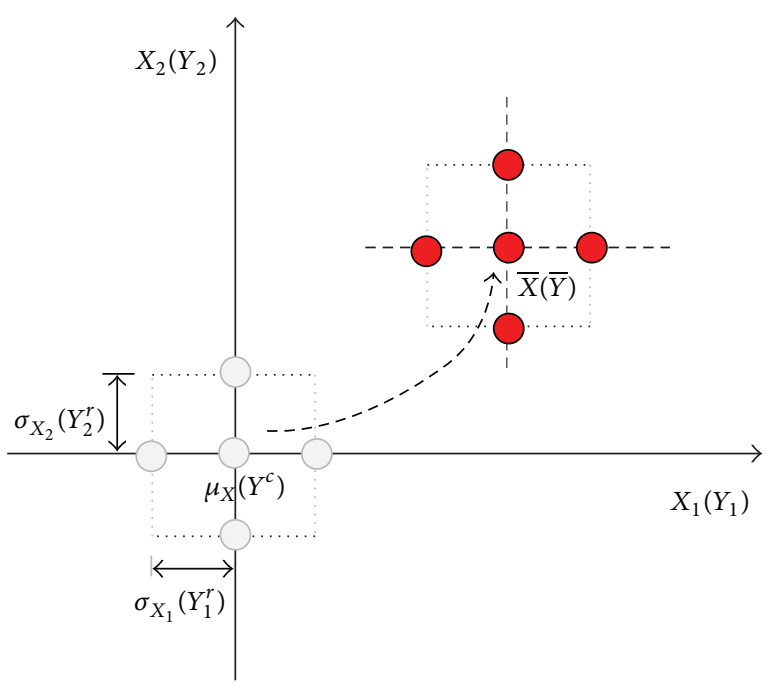

The initial sample points

New sample points

FIGURE 2: Processing of the sample points selected.

Assume that $U^{(k)}$ and $Y^{(k)}$ are obtained in the iteration process of the $k$ th step, interval variable $Y^{(k)}$ is fixed in the next step, and $U^{(k+1)}$ is calculated; namely,

$$
U^{(k+1)}=U^{(k)}+\lambda d^{(k)}
$$

where the searching direction is expressed by

$$
\begin{gathered}
d^{(k)=} \frac{\nabla \widetilde{G}\left(U^{(k)}, Y^{(k)}\right)\left(U^{(k)}\right)^{T}-\widetilde{G}\left(U^{(k)}, Y^{(k)}\right)}{\left\|\nabla \widetilde{G}\left(U^{(k)}, Y^{(k)}\right)\right\|^{2}} \\
\cdot \nabla \widetilde{G}\left(U^{(k)}, Y^{(k)}\right)-U^{(k)},
\end{gathered}
$$

where $\nabla \widetilde{G}$ is the gradient of $\widetilde{G}$, which is determined by the following function:

$$
\mathrm{m}(U, Y)=\frac{1}{2}\|U\|+c\|\widetilde{G}(U, Y)\|,
$$

where $c$ is a constant that satisfies $c>\|U\| /\left\|\nabla \widetilde{G}\left(U^{(k)}, Y^{(k)}\right)\right\|$ and $c=2\left\|U^{(k)}\right\| /\left\|\nabla \widetilde{G}\left(U^{(k)}, Y^{(k)}\right)\right\|+10 . \lambda$ is the iteration step length, which is determined by

$$
\begin{aligned}
\lambda & =s^{h} \quad(s=0.5), \\
h & =\max \left\{s^{h} \mid m\left(U^{(k)}+s^{h} d^{(k)}, Y^{(k)}\right)-m\left(U^{(k)}, Y^{(k)}\right)\right. \\
& <0\} .
\end{aligned}
$$

Then, $Y^{(k+1)}$ is calculated using interval analysis after obtaining $U^{(k+1)}$ :

$$
\begin{aligned}
Y^{(k+1)}=\min _{Y} & \widetilde{G}\left(U^{(k+1)}, Y^{(k)}\right) \\
\text { s.t. } & Y^{L} \leq Y^{(k)} \leq Y^{R}
\end{aligned}
$$




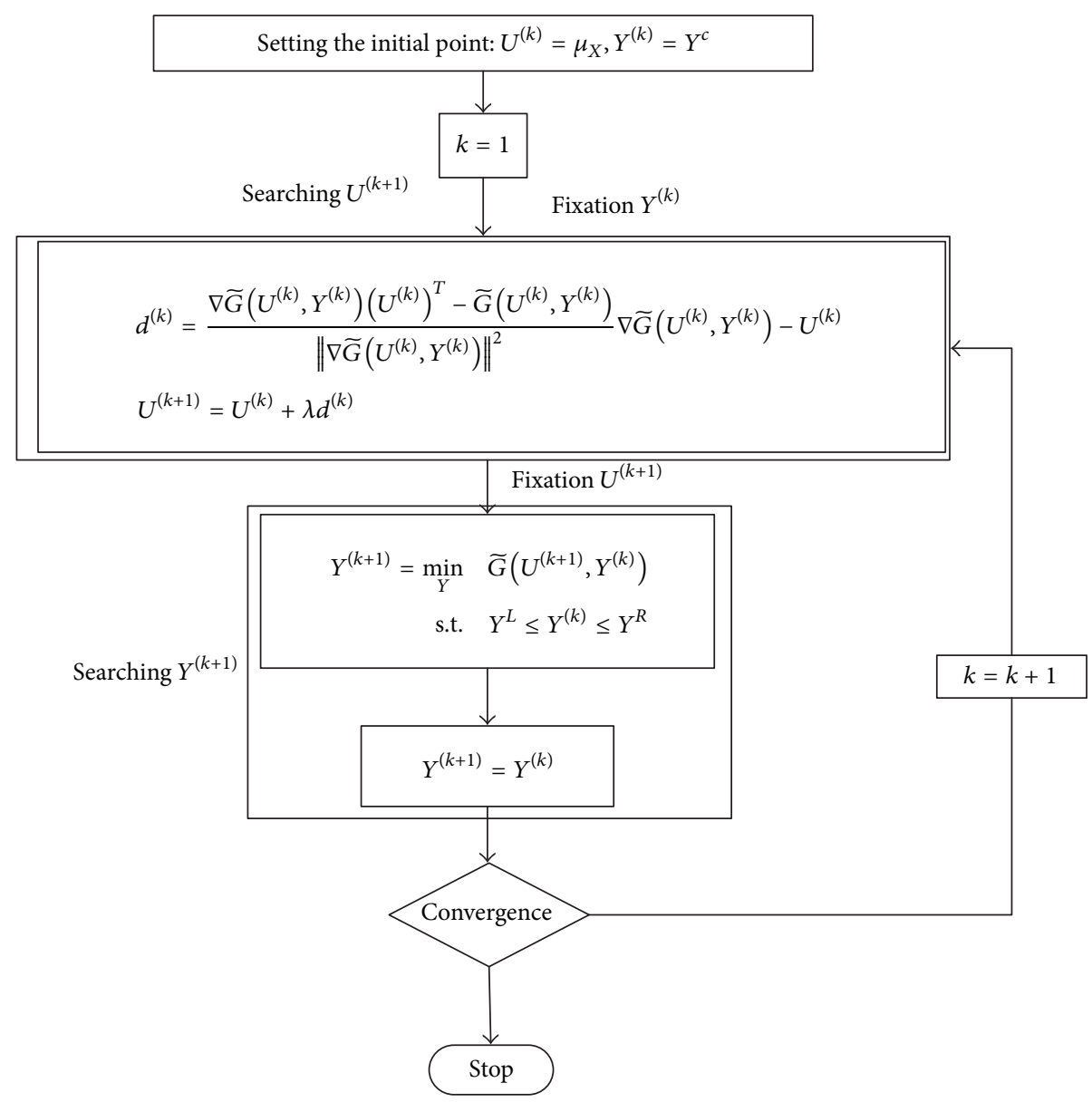

Figure 3: Flow chart of decoupling method.

until it meets

$$
\begin{gathered}
\frac{\left\|U^{(k+1)}-U^{(k)}\right\|}{\left\|U^{(k)}\right\|} \leq \varepsilon_{1}, \\
\widetilde{G}\left(U^{(k+1)}, Y^{(k+1)}\right) \leq \varepsilon_{2},
\end{gathered}
$$

where $\varepsilon_{1}$ and $\varepsilon_{2}$ are arbitrary small numbers.

The design test point $\left(\widetilde{X}^{*}, \widetilde{Y}^{*}\right)$ is obtained from the flow chart shown in Figure 3.

The design test point $\left(\widetilde{X}^{*}, \widetilde{Y}^{*}\right)$ is obtained by HRA and (7) in each iteration step. Then, the sample centre point $(\bar{X}, \bar{Y})$ that is closer to the failure surface is obtained through interpolation, as shown in Figure 4.

The expressions of the connective points $\left(\mu_{X}, Y^{c}\right.$, $\left.g\left(\mu_{X}, Y^{c}\right)\right)$ and $\left(\widetilde{X}^{*}, \widetilde{Y}^{*}, g\left(\widetilde{X}^{*}, \widetilde{Y}^{*}\right)\right)$ are obtained by

$$
\begin{aligned}
& \frac{g(X, Y)-g\left(\mu_{X}, Y^{c}\right)}{X-\mu_{X}}=\frac{g\left(\widetilde{X}^{*}, \widetilde{Y}^{*}\right)-g(X, Y)}{\widetilde{X}^{*}-X}, \\
& \frac{g(X, Y)-g\left(\mu_{X}, Y^{c}\right)}{Y-Y^{c}}=\frac{g\left(\widetilde{X}^{*}, \widetilde{Y}^{*}\right)-g(X, Y)}{\widetilde{Y}^{*}-Y} .
\end{aligned}
$$

When $g(X, Y)=0$, the design points close to the real limit state surface are acquired as follows:

$$
\begin{gathered}
\bar{X}=\mu_{X}+\left(\widetilde{X}^{*}-\mu_{X}\right) \frac{g\left(\mu_{X}, Y^{c}\right)}{g\left(\mu_{X}, Y^{c}\right)-g\left(\widetilde{X}^{*}, \widetilde{Y}^{*}\right)}, \\
Y^{\prime}=Y^{c}+\left(\widetilde{Y}^{*}-Y^{c}\right) \frac{g\left(\mu_{X}, Y^{c}\right)}{g\left(\mu_{X}, Y^{c}\right)-g\left(\widetilde{X}^{*}, \widetilde{Y}^{*}\right)} .
\end{gathered}
$$

The interval variable of the most recently obtained $Y^{\prime}$ may overflow the boundary of the interval in the process of each iteration, and, in this case, $\bar{Y}$ can be expressed as

$$
\begin{array}{ll}
\bar{Y}=\min \left(Y^{\prime}, Y^{R}\right), & \text { if } Y^{\prime}>Y^{R}, \\
\bar{Y}=\max \left(Y^{\prime}, Y^{L}\right), & \text { if } Y^{\prime}<Y^{R} .
\end{array}
$$

Based on the above analysis, the probabilistic and nonprobabilistic HRA process are as follows:

(1) Set the initial iteration point $X^{(t)}=\left(\mu_{X_{1}}, \mu_{X_{2}}, \ldots\right.$, $\left.\mu_{X_{m}}\right), Y(t)=\left(Y_{1}^{c}, Y_{2}^{c}, \ldots, Y_{n}^{c}\right)$. 


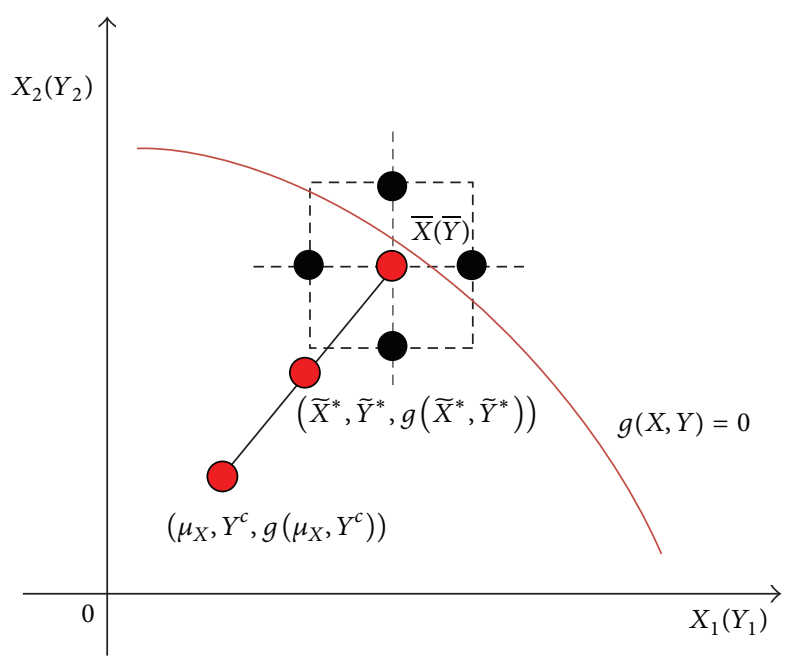

FIGURE 4: Obtaining a new sample centre point.
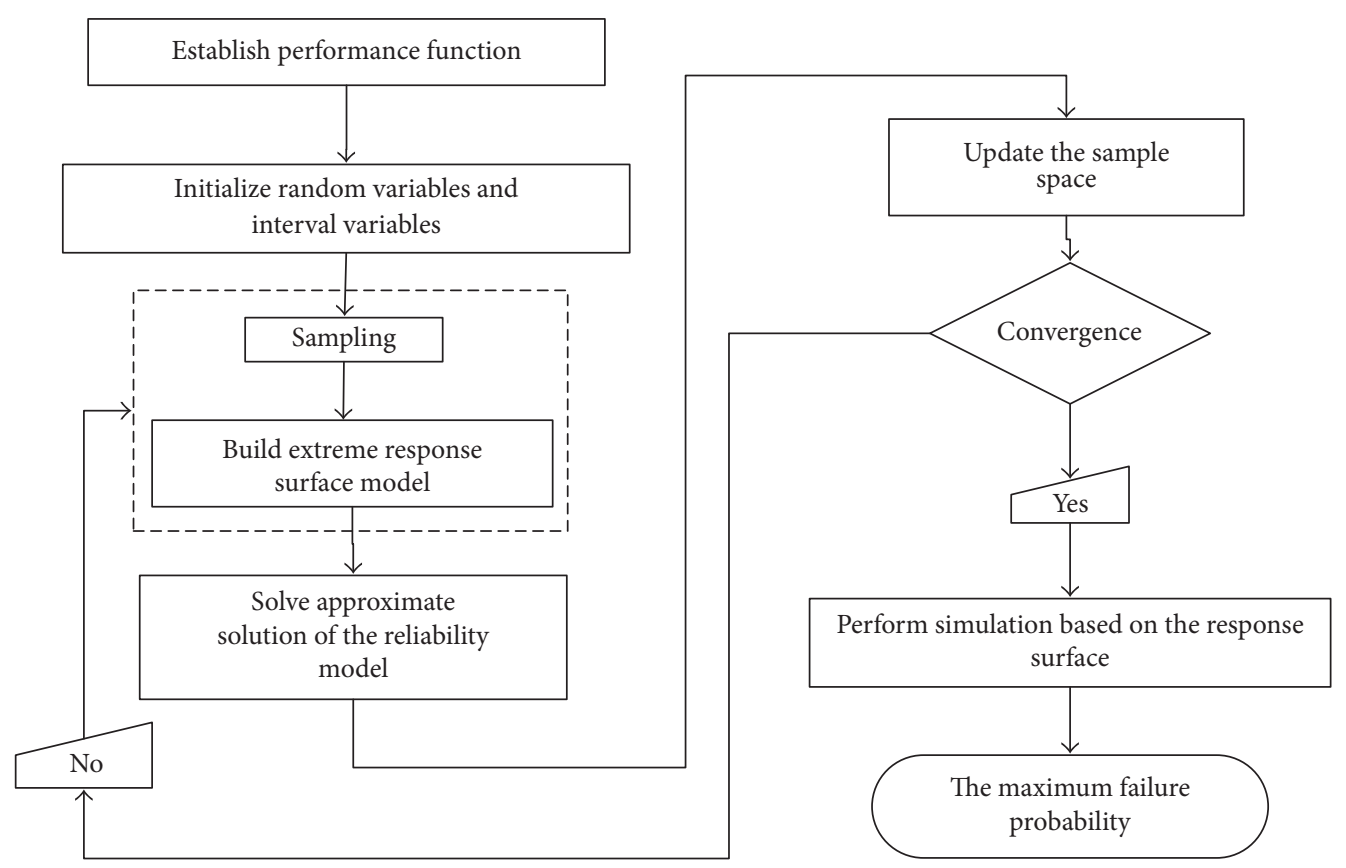

FIGURE 5: Flow chart of hybrid reliability analysis based on the extreme response surface.

(2) Establish a quadratic polynomial extremum response surface function. The sample centre point is set to $\left(\mu_{X}, Y^{c}\right)$ in the initial iteration; then, calculate the function values of $(2 m+2 n+1)$ points, and obtain the undetermined response surface coefficients.

(3) Solve the approximate mixed reliability problems using (7), and obtain the test point $\left(\widetilde{X}^{*}, \widetilde{Y}^{*}\right)$.

(4) Solve the new sample centre point $\left(\bar{X}^{(t+1)}, \bar{Y}^{(t+1)}\right)$.

(5) Judge convergence. If $\left\|\bar{X}^{(t+1)}-\bar{X}^{(t)}\right\| /\left\|\bar{X}^{(t)}\right\| \leq \varepsilon_{1}$, go to step (6); otherwise, set $t=t+1$, and go to step (2).
(6) Simulate the constructed response surface function, and calculate the maximum failure probability.

The maximum failure probability of the probabilistic and nonprobabilistic HRA can be obtained, as shown in Figure 5. To contain both random variable and interval variable analysis, MLNA is usually adopted in the conventional research, which is time-consuming and has a low computational efficiency. Nevertheless, the P-NP-HRA-DS-ERSDM is utilized and the interval analysis is embedded in the most likely failure point in the iterative process. Probabilistic analysis and nonprobabilistic analysis are investigated alternately, so 
TABLE 1: Distribution of nondeterministic variables of tuned blisk.

\begin{tabular}{lccc}
\hline Nondeterministic variables & Parameter 1 & Parameter 2 & Distribution pattern \\
\hline$w / \mathrm{rad} \cdot \mathrm{s}^{-1}$ & 1046 & 31.38 & Normal distribution \\
$t /{ }^{\circ} \mathrm{C}$ & 1050 & 31.50 & Normal distribution \\
$a / \times 10^{-5} /{ }^{\circ} \mathrm{C}^{-1}$ & 1.216 & 0.03648 & Normal distribution \\
$\mathrm{k} / \mathrm{W} \cdot\left(\mathrm{m} \cdot{ }^{\circ} \mathrm{C}\right)^{-1}$ & 27.21 & 0.8163 & Normal distribution \\
$h / \mathrm{mm}$ & 3.0 & 0.09 & Normal distribution \\
$e / \times 10^{11} / \mathrm{Pa}$ & 1.75 & 1.94 & Interval variable \\
$\mathrm{pr}$ & 0.2915 & 0.3325 & Interval variable \\
$\mathrm{den} / \mathrm{kg} \cdot \mathrm{m}^{-3}$ & 8090 & 9005 & Interval variable \\
\hline
\end{tabular}

Note. In the normal distribution, parameter 1 and parameter 2 represent the mean and the standard deviation of a random variable. In the interval variable, parameter 1 and parameter 2 represent the lower bound and upper bound. (The meaning in Table 3 is the same as in Table 1.)

TABLE 2: Analysis results of modal and vibration response of P-NP-HRA-DS-ERSDM for the tuned blisk.

(a)

\begin{tabular}{|c|c|c|c|}
\hline \multicolumn{2}{|c|}{ Random variable } & \multicolumn{2}{|c|}{ Interval variable } \\
\hline$w$ & 1129.3 & $e \times 10^{11}$ & 1.931 \\
\hline$t$ & 943.2 & den & 8072.9 \\
\hline$k$ & 29.14 & $\mathrm{pr}$ & 0.297 \\
\hline$h$ & 2.827 & - & - \\
\hline$a \times 10^{-5}$ & 1.339 & - & - \\
\hline- & - & & \\
\hline
\end{tabular}

(b)

\begin{tabular}{|c|c|c|c|c|c|c|c|c|c|c|c|}
\hline \multicolumn{7}{|c|}{ Response } & \multirow{2}{*}{\multicolumn{5}{|c|}{$\begin{array}{l}\text { The maximum failure probability } \\
\text { P-NP-HRA-DS-ERSDM }\end{array}$}} \\
\hline- & & & & & & & & & & & \\
\hline- & $P_{f \mathrm{MCS}}^{\max } / \%$ & $t /(\mathrm{h})$ & $P_{f}^{\max } / \%$ & $t /(\mathrm{h})$ & $\mathrm{Er} / \%$ & $\eta_{\mathrm{MC}} / \%$ & $P_{f}^{\max } / \%$ & $t /(\mathrm{h})$ & Er $/ \%$ & $\eta_{\mathrm{MC}} / \%$ & $\eta_{\mathrm{ML}} / \%$ \\
\hline $\begin{array}{l}f \\
2611.8 \mathrm{HZ}\end{array}$ & 98.32 & & 99.07 & & 0.76 & & 99.15 & & 0.84 & & \\
\hline dsum & 97.68 & & 99.17 & & 1.52 & & 99.26 & & 1.61 & & \\
\hline $\begin{array}{l}7.2114 \\
\text { strs }\left(\times 10^{13}\right) \\
1.4218\end{array}$ & 98.17 & 289.41 & 99.32 & 65.56 & 1.17 & 77.34 & 99.33 & 51.98 & 1.18 & 82.03 & 20.71 \\
\hline $\begin{array}{l}\text { str_e }\left(\times 10^{7}\right) \\
7.3512\end{array}$ & 98.54 & & 98.94 & & 0.41 & & 99.12 & & 0.59 & & \\
\hline $\begin{array}{l}d_{y} \\
4.12\end{array}$ & 98.16 & 297.86 & 99.12 & 77.23 & 0.97 & 74.07 & 99.76 & 62.54 & 1.62 & 79.01 & 19.02 \\
\hline
\end{tabular}

Note. $P_{f M C S}^{\max }$ : the maximum failure probability using MCS, $t$ : computing time, Er: relative error to the MCM, $\eta_{\mathrm{MC}}$ : relative computational efficiency to MCM, and $\eta_{\mathrm{ML}}$ : relative computational efficiency to MLNA. (The meaning in Table 4 is the same as in Table 2.)

the intermediate values can call each other in turn, and the computational efficiency is improved, observably.

\section{P-NP-HRA of Blisk}

P-NP-HRA-DS-ERSDM is used to analyse a blisk and is compared with MLNA and MCS to verify its scientific rationality.

3.1. P-NP-HRA of a Tuned Blisk. First, the reliability of the tuned blisk is investigated, and the natural frequency, modal shape, and vibration response are studied using P-NP-HRADS-ERSDM.
In the research, the rotational speed $w$, gas temperature $t$, blade thickness $h$, expansion coefficient $a$, and thermal conductivity coefficient $k$ are regarded as random variables, and all of them are assumed to obey the normal distribution and to be independent of each other. The elastic modulus $e$, density den, and Poisson's ratio (pr) are regarded as interval variables. Their distribution types and parameters are shown in Table 1, and the results of the calculation are shown in Table 2 .

The maximum failure probabilities of the natural frequency, modal shape, and vibration response for the tuned blisk are investigated using MCM, MLNA, and P-NPHRA-DS-ERSDM, respectively. The relative errors and the 
TABLE 3: Distribution of nondeterministic variables of mistuned blisk.

\begin{tabular}{lccr}
\hline Nondeterministic variables & Parameter 1 & Parameter 2 & Distribution pattern \\
\hline$w / \mathrm{rad} \cdot \mathrm{s}^{-1}$ & 1046 & 31.38 & Normal distribution \\
$t /{ }^{\circ} \mathrm{C}$ & 1050 & 31.50 & Normal distribution \\
$h / \mathrm{mm}$ & 3.0 & 0.09 & Normal distribution \\
$\mathrm{pr}_{b}$ & 0.3181 & 0.009542 & Normal distribution \\
$a_{b} / \times 10^{-5} /{ }^{\circ} \mathrm{C}^{-1}$ & 1.268 & 0.03806 & Normal distribution \\
$k_{b} / \mathrm{W} \cdot\left(\mathrm{m} \cdot{ }^{\circ} \mathrm{C}\right)^{-1}$ & 29.72 & 0.8915 & Normal distribution \\
$\mathrm{den}_{b}$ & 8010 & 8943 & Interval variable \\
$e_{b} \times 10^{11} / \mathrm{Pa}$ & 1.689 & 1.932 & Interval variable \\
$\mathrm{pr}_{d}$ & 0.3143 & 0.009429 & Normal distribution \\
$a_{d} / \times 10^{-5} /{ }^{\circ} \mathrm{C}$ & 0.03648 & Normal distribution \\
$k_{d} / \mathrm{W} \cdot\left(\mathrm{m} \cdot{ }^{\circ} \mathrm{C}\right)^{-1}$ & 1.216 & 0.8163 & Normal distribution \\
$\operatorname{den}_{d}$ & 27.21 & 9280 & Interval variable \\
$e_{d} \times 10^{11} / \mathrm{Pa} \times 10^{11} / \mathrm{Pa}$ & 8170 & 1.996 & Interval variable \\
\hline
\end{tabular}

computational efficiency of all three methods are analysed, as shown in Table 2. For the natural frequency, modal displacement, modal stress, modal strain energy, and vibration response, the relative errors of MLNA to MCM are $0.76 \%, 1.52 \%, 1.17 \%, 0.41 \%$, and $0.97 \%$, respectively, and the relative errors of P-NP-HRA-DS-ERSDM to MCM are $0.84 \%$, $1.61 \%, 1.18 \%, 0.59 \%$, and $1.62 \%$, respectively; these values meet the engineering requirements. For the calculation of modal and vibration response, the computational efficiency of MLNA relative to MCM increases by $77.34 \%$ and $74.07 \%$, respectively, and the computational efficiency of P-NP-HRADS-ERSDM relative to MCM increases by $82.03 \%$ and $79.01 \%$, respectively. Thus, the computational efficiency of P-NPHRA-DS-ERSDM relative to MLNA increased by $20.71 \%$ and $19.02 \%$, respectively. Therefore, the computational accuracy of using P-NP-HRA-DS-ERSDM and MLNA approximately equals that of using MCM. However, the computational efficiency of P-NP-HRA-DS-ERSDM is higher than that of MLNA.

3.2. P-NP-HRA of a Mistuned Blisk. For a mistuned blisk, the rotational speed $w$, gas temperature $t$, blade thickness $h$, Poisson's ratio of the blade $\mathrm{pr}_{b}$, expansion coefficient of the blade $a_{b}$, thermal conductivity coefficient of the blade $k_{b}$, Poisson's ratio of the disk $\mathrm{pr}_{d}$, expansion coefficient of the disk $a_{d}$, and thermal conductivity coefficient of the disk $k_{d}$ are regarded as random variables, and all of them are hypothesized to obey the normal distribution and to be independent of each other. The elastic modulus of the blade

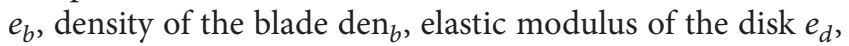

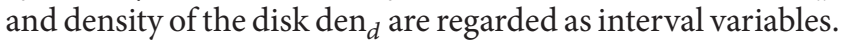
Their distribution types and parameters are shown in Table 3, and the calculation results are shown in Table 4.

The maximum failure probability of the natural frequency, modal shape, and vibration response for the mistuned blisk are calculated using MCM, MLNA, and P-NPHRA-DS-ERSDM. The relative errors and the computational efficiency of the three methods are analysed, as shown in Table 4. When the blisk is mistuned, the computational time is very long using MCM, and convergence may not be achieved. The computational accuracy of P-NP-HRA-DSERSDM approximately equals that of MLNA, while the computational efficiency of P-NP-HRA-DS-ERSDM is $22.86 \%$ and $25.18 \%$ higher than that of MLNA. The increase in computational efficiency for P-NP-HRA-DS-ERSDM compared to MLNA is higher for the mistuned blisk than for the tuned blisk. Furthermore, the superiority of this methodology is more obvious for the mistuned blisk than for the tuned blisk, verifying that this method is feasible.

\section{Conclusions}

A nondeterministic analysis method with high efficiency and high accuracy, P-NP-HRA-DS-ERSDM, is investigated for a blisk. In the analysis process, for the hybrid nondeterministic problem containing both random variables and interval variables, the interval failure probability and interval reliability index are obtained. A flow chart is presented for solving the reliability analysis and the extremum response surface HRA using P-NP-HRA-DS-ERSDM.

Probabilistic and nonprobabilistic HRA is used to analyse the blisk. The variables mainly affecting the output response are regarded as interval variables, and the other variables are regarded as random variables. In the tuned blisk, $e$, den, and $\mathrm{pr}$ are regarded as interval variables, while $w, t, h, a$, and $k$ are regarded as random variables. However, in the mistuned blisk, den $n_{b}, e_{b}$, den ${ }_{d}$, and $e_{d}$ are regarded as interval variables, while $w, t, h, \mathrm{pr}_{b}, a_{b}, k_{b}, \mathrm{pr}_{d}, a_{d}$, and $k_{d}$ are regarded as random variables.

The maximum failure probabilities of the natural frequency, modal displacement, modal stress, modal strain energy, and vibration response are calculated for blisk, comparing the computational time and the relative error of P-NP-HRA-DS-ERSDM, MCM, and MLNA. For the tuned blisk, the relative errors of the natural frequency, modal displacement, modal stress, modal strain energy, and vibration response using MLNA to MCM are $0.76 \%$, $1.52 \%, 1.17 \%, 0.41 \%$, and $0.97 \%$, respectively, and those of using P-NP-HRA-DS-ERSDM are 0.84\%, 1.61\%, 1.18\%, 0.59\%, and $1.62 \%$; these values meet the engineering requirements. 
TABLE 4: Analysis results of modal and vibration response of P-NP-HRA-DS-ERSDM for the mistuned blisk.

(a)

\begin{tabular}{|c|c|c|c|}
\hline \multicolumn{2}{|c|}{ Random variable } & \multicolumn{2}{|c|}{ Interval variable } \\
\hline$w$ & 1129.3 & $\operatorname{den}_{d}$ & 8072.9 \\
\hline$t$ & 943.2 & $\operatorname{den}_{b}$ & 7977.8 \\
\hline$k_{d}$ & 29.14 & $e_{d} \times 10^{11}$ & 1.931 \\
\hline$k_{b}$ & 27.63 & $e_{b} \times 10^{11}$ & 1.889 \\
\hline $\mathrm{pr}_{d}$ & 0.297 & & \\
\hline $\mathrm{pr}_{b}$ & 0.294 & & \\
\hline$h$ & 2.827 & - & - \\
\hline$a_{b} \times 10^{-5}$ & 1.251 & & \\
\hline$a_{d} \times 10^{-5}$ & 1.339 & & \\
\hline
\end{tabular}

(b)

\begin{tabular}{|c|c|c|c|c|c|c|c|c|c|c|c|}
\hline \multirow{3}{*}{$\begin{array}{l}\text { Response } \\
- \\
- \\
\end{array}$} & \multicolumn{11}{|c|}{ The maximum failure probability } \\
\hline & \multicolumn{2}{|c|}{ MCM } & \multicolumn{4}{|c|}{ MLNA } & \multicolumn{5}{|c|}{ P-NP-HRA-DS-ERSDM } \\
\hline & $P_{f \mathrm{MCS}}^{\max } / \%$ & $t /(h)$ & $P_{f}^{\max } / \%$ & $t /(h)$ & $\mathrm{Er} / \%$ & $\eta_{\mathrm{MC}} / \%$ & $P_{f}^{\max } / \%$ & $t /(h)$ & $\mathrm{Er} / \%$ & $\eta_{\mathrm{MC}} / \%$ & $\eta_{\mathrm{ML}} / \%$ \\
\hline $\begin{array}{l}f \\
2611.8 \mathrm{HZ}\end{array}$ & & & 99.17 & & & & 99.87 & & & & \\
\hline dsum & & & 99.37 & & & & 99.31 & & & & \\
\hline $\begin{array}{l}20.943 \\
\operatorname{strs}\left(\times 10^{13}\right) \\
3.6745\end{array}$ & - & - & 99.46 & 289.21 & - & - & 99.32 & 223.09 & - & - & 22.86 \\
\hline $\begin{array}{l}\text { str_e }\left(\times 10^{7}\right) \\
372.84\end{array}$ & & & 98.89 & & & & 99.24 & & & & \\
\hline $\begin{array}{l}d_{y} \\
6.56\end{array}$ & - & - & 99.21 & 312.56 & - & - & 99.86 & 231.59 & - & - & 25.18 \\
\hline
\end{tabular}

The computational efficiency of MLNA relative to MCM increases by $77.34 \%$ and $74.07 \%$, respectively, but that of P-NP-HRA-DS-ERSDM increases by $82.03 \%$ and $79.01 \%$, respectively. Thus, the computational efficiency of P-NPHRA-DS-ERSDM relative to MLNA increased by $20.71 \%$ and $19.02 \%$. However, for the mistuned blisk, the computational efficiency of P-NP-HRA-DS-ERSDM increases by $22.86 \%$ and $25.18 \%$ higher than that of MLNA, and the computational time is very long using MCM, and the convergence may not be achieved, which manifests that the superiority of this methodology is more obvious for the mistuned blisk than for the tuned blisk. The scientific rationality and validity for researching a blisk using P-NP-HRA-DS-ERSDM is verified, and this method is shown to be particularly superior to MLNA for a mistuned blisk.

\section{Conflicts of Interest}

The author declares that there are no conflicts of interest regarding the publication of this paper.

\section{Acknowledgments}

This work has been supported by the National Natural Science Foundation of China (Grant no. 51375032) and
Project supported by Beijing Postdoctoral Research Foundation (Grant no. 2016ZZ-12).

\section{References}

[1] Y. Ben-Haim, "A non-probabilistic concept of reliability," Structural Safety, vol. 14, no. 4, pp. 227-245, 1994.

[2] I. Elishakoff, "Essay on uncertainties in elastic and viscoelastic structures: from A. M. Freudenthal's criticisms to modern convex modeling," Computers and Structures, vol. 56, no. 6, pp. 871-895, 1995.

[3] Y. Ben-Haim and I. Elishakoff, "Discussion on: a non-probabilistic concept of reliability," Structural Safety, vol. 17, no. 3, pp. 195-199, 1995.

[4] Z. P. Qiu, D. Yang, and I. Elishakoff, "Combination of structural reliability and interval analysis," Acta Mechanica Sinica, vol. 24, no. 1, pp. 61-67, 2008.

[5] Z. P. Qiu and J. Wang, "The interval estimation of reliability for probabilistic and non-probabilistic hybrid structural system," Engineering Failure Analysis, vol. 17, no. 5, pp. 1142-1154, 2010.

[6] Z. Ni and Z. P. Qiu, "Hybrid probabilistic fuzzy and nonprobabilistic model of structural reliability," Computers and Industrial Engineering, vol. 58, no. 3, pp. 463-467, 2010.

[7] J. Wang and Z. P. Qiu, "Fatigue reliability based on residual strength model with hybrid uncertain parameters," Acta Mechanica Sinica, vol. 28, no. 1, pp. 112-117, 2012. 
[8] P. Fang, X. L. Chang, K. Hu, Z. X. Wang, and B. Long, “The hybrid probabilistic and non-probabilistic model of structural reliability analysis," in Proceedings of the 1st Symposium on Aviation Maintenance and Management. Vol II, vol. 297, pp. 237244, Xian, China, 2014.

[9] J. Wang and Z. P. Qiu, "The reliability analysis of probabilistic and interval hybrid structural system," Applied Mathematical Modelling, vol. 34, no. 11, pp. 3648-3658, 2010.

[10] X. J. Wang, L. Wang, and Z. P. Qiu, "Safety estimation of structural systems via interval analysis," Chinese Journal of Aeronautics, vol. 26, no. 3, pp. 614-623, 2013.

[11] C. Wang, Z. P. Qiu, and Y. Y. He, "Fuzzy interval perturbation method for uncertain heat conduction problem with interval and fuzzy parameters," International Journal for Numerical Methods in Engineering, vol. 104, no. 5, pp. 330-346, 2015.

[12] H. An, L. Zhou, and H. Sun, "Structural hybrid reliability index and its convergent solving method based on random-fuzzyinterval reliability model," Advances in Mechanical Engineering, vol. 8 , no. 8, pp. 1-13, 2016.

[13] C. Jiang, X. Han, and G. Y. Lu, "A hybrid reliability model for structures with truncated probability distributions," Acta Mechanica, vol. 223, no. 9, pp. 2021-2038, 2012.

[14] C. Jiang, X. Y. Long, X. Han, Y. R. Tao, and J. Liu, "Probabilityinterval hybrid reliability analysis for cracked structures existing epistemic uncertainty," Engineering Fracture Mechanics, vol. 112-113, pp. 148-164, 2013.

[15] C. Jiang, J. Zheng, B. Y. Ni, and X. Han, "A probabilistic and interval hybrid reliability analysis method for structures with correlated uncertain parameters," International Journal of Computational Methods, vol. 12, no. 4, Article ID 1540006, 2015.

[16] X. Han, C. Jiang, L. X. Liu, J. Liu, and X. Y. Long, "Responsesurface-based structural reliability analysis with random and interval mixed uncertainties," Science China Technological Sciences, vol. 57, no. 7, pp. 1322-1334, 2014.

[17] L. Wang, X. J. Wang, and Y. Xia, "Hybrid reliability analysis of structures with multi-source uncertainties," Acta Mechanica, vol. 225, no. 2, pp. 413-430, 2014.

[18] J. E. Hurtado and D. A. Alvarez, "The encounter of interval and probabilistic approaches to structural reliability at the design point," Computer Methods in Applied Mechanics and Engineering, vol. 225-228, pp. 74-94, 2012.

[19] W. Gao, D. Wu, C. M. Song, F. Tin-Loi, and X. J. Li, "Hybrid probabilistic interval analysis of bar structures with uncertainty using a mixed perturbation Monte-Carlo method," Finite Elements in Analysis and Design, vol. 47, no. 7, pp. 643-652, 2011.

[20] S. S. Cao and J. Q. Lei, "Hybrid reliability model for fatigue reliability analysis of steel bridges," Journal of Central South University, vol. 23, no. 2, pp. 449-460, 2016.

[21] D. Wu, W. Gao, C. M. Song, and S. Tangaramvong, "Probabilistic interval stability assessment for structures with mixed uncertainty," Structural Safety, vol. 58, pp. 105-118, 2016.

[22] H. X. Xue, W. Y. Tang, S. K. Zhang, and M. Yuan, "Interval analysis method of fatigue and fracture reliability for offshore structures based on probabilistic and non-probabilistic hybrid model," in Proceedings of the 16th International Offshore and Polar Engineering Conference (ISOPE '06), pp. 370-375, San Francisco, Calif, USA, June 2006.

[23] Z. T. Zhao, J. B. Guo, and S. K. Zeng, “The method of dynamic systems reliability modeling based on hybrid theory and interval analysis," in Proceedings of the 1st International Conference on Reliability Systems Engineering (ICRSE '15), Beijing, China, October 2015.
[24] C. Ibáñez-Llano, A. Rauzy, E. Meléndez, and F. Nieto, "Hybrid approach for the assessment of PSA models by means of binary decision diagrams," Reliability Engineering and System Safety, vol. 95, no. 10, pp. 1076-1092, 2010.

[25] Y. J. Luo, Z. Kang, and A. Li, "Structural reliability assessment based on probability and convex set mixed model," Computers and Structures, vol. 87, no. 21-22, pp. 1408-1415, 2009.

[26] Y. J. Luo, A. Li, and Z. Kang, "Reliability-based design optimization of adhesive bonded steel-concrete composite beams with probabilistic and non-probabilistic uncertainties," Engineering Structures, vol. 33, no. 7, pp. 2110-2119, 2011.

[27] N. Pedroni and E. Zio, "Hybrid uncertainty and sensitivity analysis of the model of a twin-jet aircraft," Journal of Aerospace Information Systems, vol. 12, no. 1, pp. 73-96, 2015.

[28] X. Liu and Z. Y. Zhang, "A hybrid reliability approach for structure optimisation based on probability and ellipsoidal convex models," Journal of Engineering Design, vol. 25, no. 46, pp. 238-258, 2014.

[29] X. F. Yang, Y. S. Liu, Y. S. Zhang, and Z. F. Yue, "Probability and convex set hybrid reliability analysis based on active learning kriging model," Applied Mathematical Modelling, vol. 39, no. 14, pp. 3954-3971, 2015.

[30] X. F. Yang, Y. S. Liu, Y. Gao, Y. S. Zhang, and Z. Z. Gao, "An active learning kriging model for hybrid reliability analysis with both random and interval variables," Structural and Multidisciplinary Optimization, vol. 51, no. 5, pp. 1003-1016, 2015.

[31] L. Wang, X. J. Wang, R. X. Wang, and X. Chen, "Reliabilitybased design optimization under mixture of random, interval and convex uncertainties," Archive of Applied Mechanics, vol. 86, no. 7, pp. 1341-1367, 2016.

[32] B. Z. Xia and D. J. Yu, "Change-of-variable interval stochastic perturbation method for hybrid uncertain structural-acoustic systems with random and interval variables," Journal of Fluids and Structures, vol. 50, pp. 461-478, 2014.

[33] N. Chen, D. J. Yu, and B. Z. Xia, "Unified analysis approach for the energy flow in coupled vibrating systems with two types of hybrid uncertain parameters," Mechanical Systems and Signal Processing, vol. 70-71, pp. 542-556, 2016.

[34] W. S. Peng, J. G. Zhang, and L. F. You, "The hybrid uncertain neural network method for mechanical reliability analysis," International Journal of Aeronautical and Space Sciences, vol. 16, no. 4, pp. 510-519, 2015.

[35] J. Fan and Y. P. Zhang, "A hybrid probability-convex model for the seismic demand analysis of bearing displacement in the benchmark base-isolated structure," Advances in Structural Engineering, vol. 17, no. 7, pp. 1061-1073, 2014.

[36] W. Zhang, J. Liu, C. Cho, and X. Han, "A hybrid parameter identification method based on Bayesian approach and interval analysis for uncertain structures," Mechanical Systems and Signal Processing, vol. 60, pp. 853-865, 2015.

[37] Y. Xu, G. H. Huang, M. F. Cao, C. Dong, and Y. F. Li, "A hybrid waste-flow allocation model considering multiple stage and interval-fuzzy chance constraints," Civil Engineering and Environmental Systems, vol. 29, no. 1, pp. 59-78, 2012.

[38] H. An and W. G. An, "An approach to fatigue reliability analysis based on the hybrid model of probability and non-probability," Advanced Materials Research, vol. 291-294, pp. 2179-2182, 2011.

[39] J. Drugowitsch and A. Pouget, "Probabilistic vs. non-probabilistic approaches to the neurobiology of perceptual decisionmaking," Current Opinion in Neurobiology, vol. 22, no. 6, pp. 963-969, 2012. 
[40] B. Bai, G. C. Bai, and C. Li, "Application of improved hybrid interface substructural component modal synthesis method in vibration characteristics of mistuned blisk," Chinese Journal of Mechanical Engineering, vol. 27, no. 6, pp. 1219-1231, 2014.

[41] X. P. Du, "Interval reliability analysis," in Proceedings of the 33rd Design Automation Conference-ASME International Design Engineering Technical Conferences and Computers and Information in Engineering Conference (IDETC/CIE '07), pp. 1103-1109, Las Vegas, Nev, USA, September 2007. 


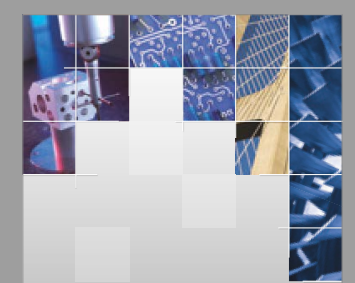

\section{Enfincering}
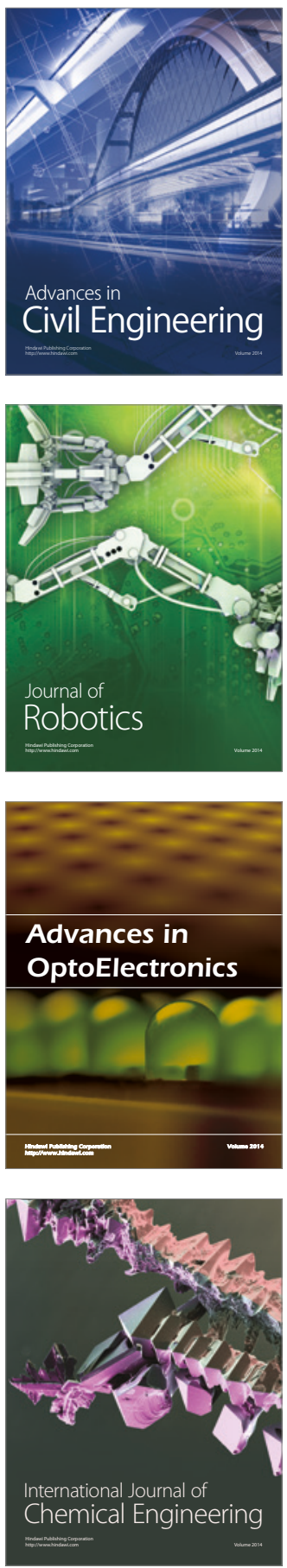

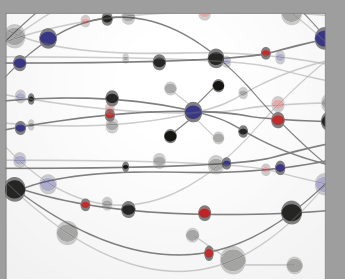

The Scientific World Journal

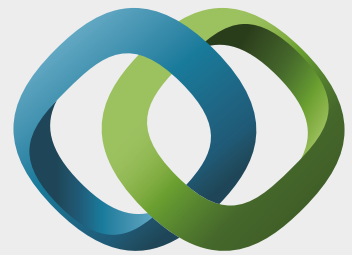

\section{Hindawi}

Submit your manuscripts at

https://www.hindawi.com
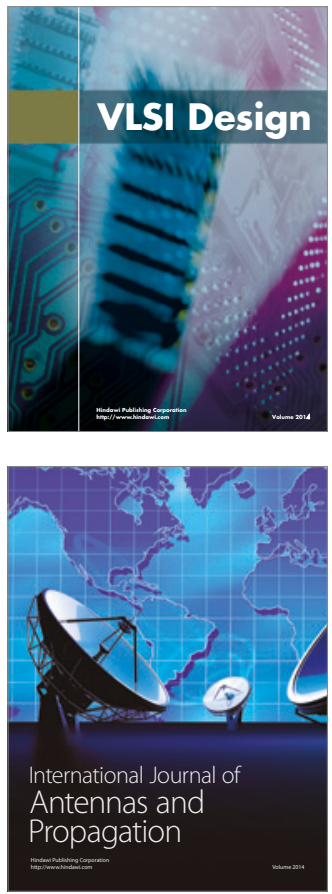

\section{Rotating}

Machinery
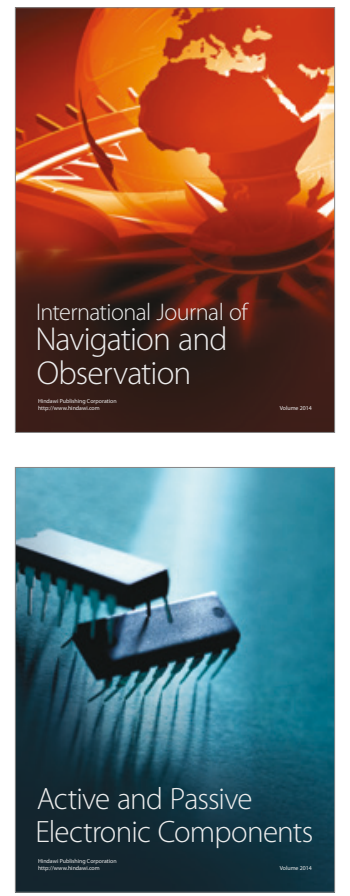
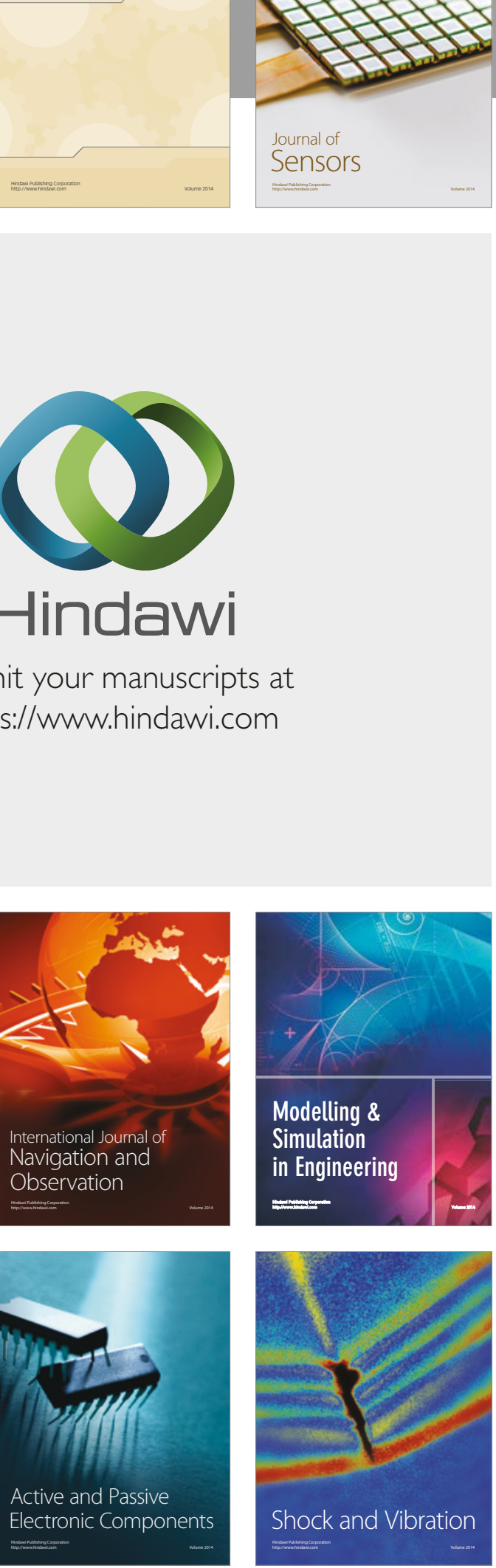
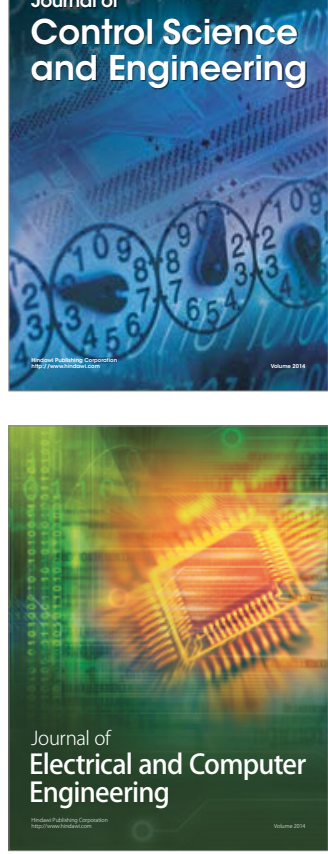

Distributed

Journal of

Control Science

and Engineering
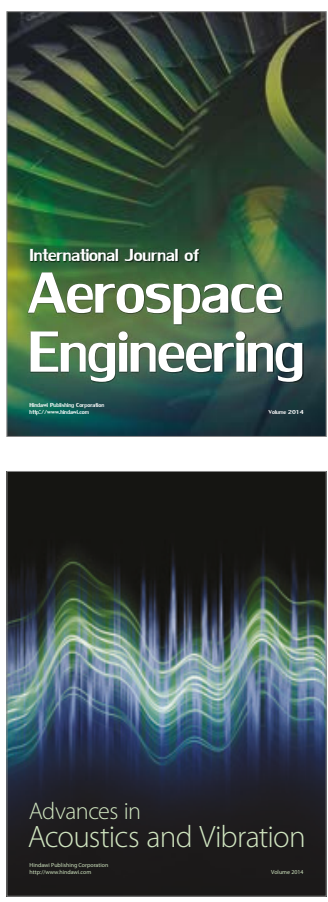

Sensor Networks 